\title{
Snake bite: Medico-legal Significance
}

\author{
Raveendran $\mathrm{S}^{1^{*}}$, Vidanapathirana $\mathrm{M}^{2}$
}

\begin{abstract}
Introduction: Most of the snake bite deaths reported were due to delay in giving antivenom or non-availability of species specific antivenom and the relatives of deceased allege against doctors that proper care is not given. Forensic pathologist is expected not only to give cause of death but also give opinions about potential negligence in such cases. Case report: A middle aged woman died while being transported from a local hospital to a tertiary care hospital following a snake bite. She was brought to the hospital with symptoms suggestive of neurotoxin poisoning and anti-snake venom was given at the local hospital. The patient developed shortness of breath and she was transferred to a tertiary-care hospital for further ventilator support, but the patient was pronounced dead on the way after 2 hours of the bite. Bite mark with two fangs was found on left upper part of the breast. Inflammatory signs were found around the bite mark with necrosis and blister formation. Autopsy revealed no hematological manifestations. Conclusions: The COD was envenomation of neurotoxin due to cobra bite. The allegation of medical negligence may be challenged, because death could occur rapidly within 30 minutes to two hours in cobra bites and delay in transport may also have played a vital role especially being a rural area. Role of non-availability of species specific antivenom and efficacy of common anti snake venom should be further studied before giving opinions regarding potential negligence.
\end{abstract}

Key words: cobra bite, breast, intubation, anti-snake venom, negligence

\section{Full paper}

\section{Introduction}

There are 2400 species of snakes distributed around the world, of them $10 \%$ are venomous [1]. Sri Lanka has only 93 species of land and marine snakes of which five (5) are deadly venomous, two (2) are moderately venomous. The majority of snakes in Sri Lanka are non-venomous [1].

Snake bites are a common problem in Sri Lanka among people living in rural and agricultural areas. Most of the deaths reported in victims with snakebites were due to delay in giving antivenom or non-availability of species-specific antivenom. Relatives of deceased allege against doctors that up front and proper care is not given. Forensic pathologist is expected not only to give cause of death but also give opinions about negligence in such cases.

${ }^{1}$ Postgraduate trainee, ${ }^{2}$ Professor, Department of Forensic Medicine, Faculty of Medical Sciences, University of Sri Jayewardenepura

*Corresponding author: S Raveendran, email: sathasivamraveendran10@gmail.com, Tel: 0094773867534

\section{Case report}

A middle-aged woman died while being transported from local hospital to tertiary care hospital following snake bite. History revealed that snake had bitten her while she was trying to get on to the bicycle, which parked in an abandoned house around 6.15 p.m. She was brought to the hospital with symptoms and signs suggestive of neurotoxin poisoning such as blurred vision, dysphonia, dysphagia and confusion with ptosis, but she had no haematemesis, haematuria, epistaxis or anal bleeding. Anti-snake venom was given at the local hospital with clinical diagnosis without dead specimen of snake. The patient developed shortness of breath while anti snake venom being infused. Clinicians had tried to intubate, but failed and was managed with ambu bag and mask ventilation. She was transferred to a tertiary-care hospital for further ventilator support and management but died before admission after 2 hours of the snakebite.

Relatives alleged that proper management was not provided even they brought the victim early to the hospital, such as failed intubation. The killed cobra with a gunshot wound was brought to the morgue on next day morning (Figure 1).

At the autopsy, original clothes were not available. Bite mark with two fang marks (Figure 2) were found on left upper and inner part of the breast. 
Inflammatory signs were found around bite mark with swelling, necrosis and blister formation (Figure $3)$. No physical injuries found to suggest any violence. Internal examination was unremarkable except pulmonary congestion and oedema. There were no hematological manifestations such as haematuria, gastro-intestinal bleeding, mucosal bleeding, etc. At the end of the examination, the COD was envenomation due to cobra bite.

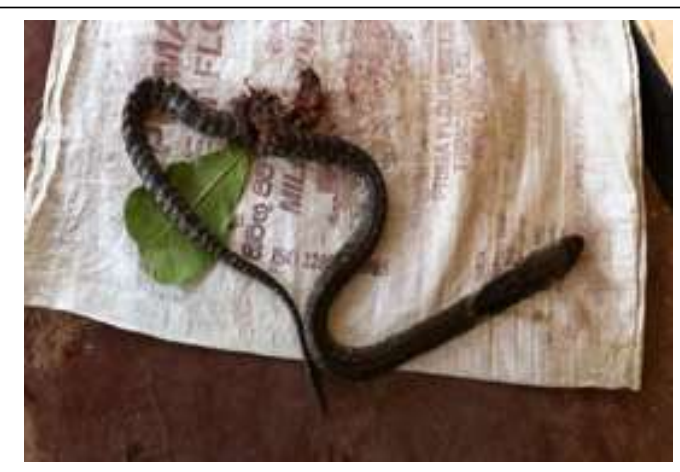

Figure 1: Specimen of killed cobra
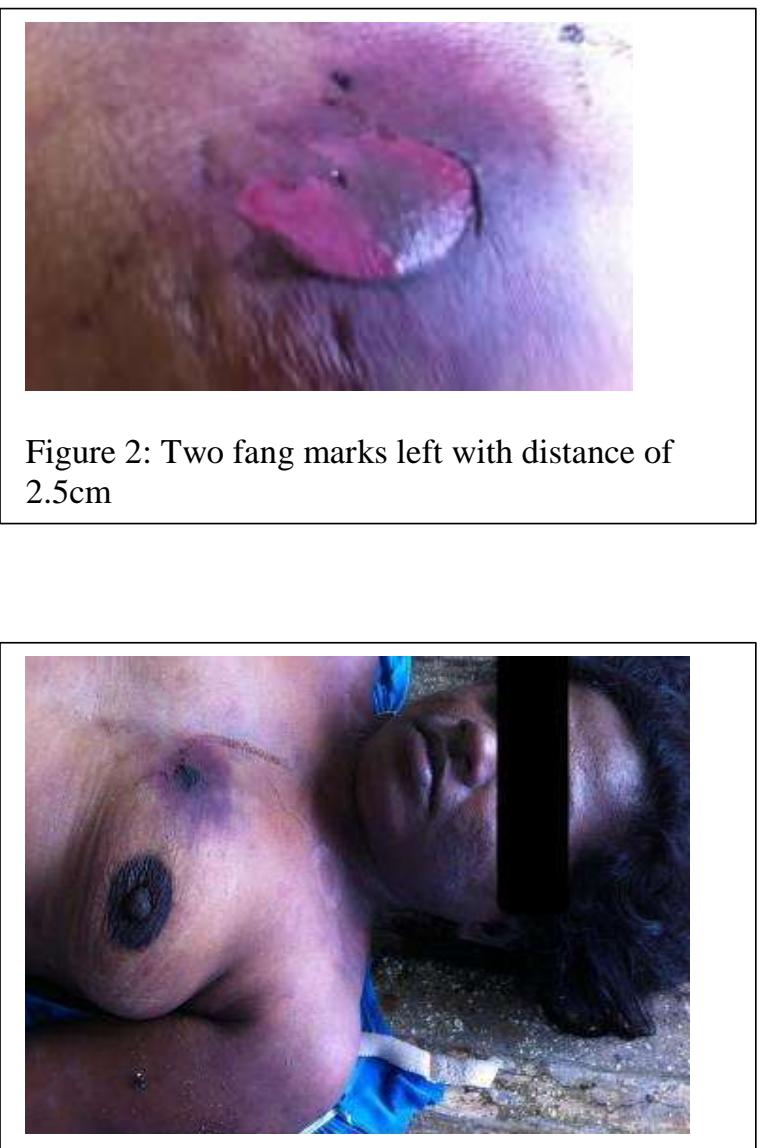

Figure 3: Site of Cobra bite with severe inflammation and local necrosis between head and body and spectacle marking on its dorsal surface) [2], severe local signs of

\section{Discussion}

Peculiar symptoms and signs of neurotoxic agent, rapid progression of death, day biting behavior of snake, place of bite near to human dwelling, morphological features of killed snake (Hood between head and body and spectacle marking on its dorsal surface) [2], severe local signs of envenomation at the site of bite and absence of natural diseases confirmed COD as envenomation with neurotoxin due to cobra bite.

Common cause of death among the snake bites in Sri Lanka is viper bite which is excluded in this case due to absence of hematological manifestations. Krait (Indian and Ceylon) usually bites at night in victims who sleeps on floor within small hut or houses in cultivated areas [3]. Krait bite usually does not produce visible fang marks or local reaction. Krait bite was further excluded due to presence of severe local signs and rapid progression to death [3].

Local signs are predominant in bite due to cobra and viper [4]. Features of neurotoxin are dominant in cobra and krait (Elapidae family) [5] and features of coagulopathy are dominant in Russell's viper, Saw scaled viper and in Hump nosed viper (Family Viperidae) [6].

Peculiar feature observed in this case was that the bite was found in breast in contrast to usual site of bite in lower limb and not commonly in upper limb [7]. This may be the reason of the quick death in this case. Absorption of venom is very quick in chest when compared with upper or lower limbs [7]. Though the original clothes were not available, the thin tight clothes are not protective in snakebite that was observed in this case.

Allegation made by relatives regarding negligence cannot be proved confidently in this case, even though there was an allegation regarding the management of respiratory failure. The clinicians had exercised reasonable degree of skills by starting anti snake venom and made all efforts to transfer the patient to a tertiary care hospital early as possible. However, failure of intubation may have been overcome by the accepted ambu and mask ventilation [2].

Place of ineffectiveness of anti-snake venom in neutralizing local envenomation in post synaptic cleft of neuromuscular junction in chest muscle might have played a vital role in causing an early death. 
Anti-snake venom usually neutralizes the systemic envenomation but not local envenomation [8].

Anti-venom therapies available in Sri Lanka are haffkine (Mumbai) and vins antivenom (Hyderabad) [9]. It is a polyvalent antibodies prepared by injecting the venom of these snakes into a hoarse. This is prepared for Indian species-specific snakes but not for Sri Lankan snakes.

There are hardly any studies on cobra venom performed in Sri Lanka to characterize the constituents [10]. However, in India a low molecular weight isoform of hyaluronidase has been isolated from Indian cobra (N. naja) venom, which has hydrolysed hyaluronan in human skin sections. Moreover, cytotoxic and myotoxic phospholipases A2 have been characterized in Indian cobra venom $[11,12]$. This knowledge could be extrapolated to the Sri Lankan context to explain the extensive local damage and its varying degree of severity from patient to patient. Clinically, an Indian and a Sri Lankan study have documented similar extensive skin necrosis in cobra bite in the past [10].

It had been established that krait venom damages presynaptic nerve ending, where recovery takes a longer time, whilst cobra venom is post-synaptic and its effect reverses fast. It is intriguing and interesting to see the contrasting behavior of neurotoxins of two elapids in the same geographical location [13].

Research done in Sri Lanka over the past several years with the assistance from United Kingdom Universities, local doctors have managed to produce a Sri Lankan anti venom called Polonga Tab. "This is very much better than the Indian anti venom tested it on patients in Anuradhapura. But it is very expensive since get this produced in UK and on sheep."

AVS should not be used inappropriately. Unfortunately, practices such as administering Haffkine AVS for hump-nosed viper bites still continue [14] and must be strongly discouraged. The average dose $(10 \mathrm{~g})$ of the presently available Haffkine polyvalent AVS costs about Rs. 8,000 per patient. The monovalent Polonga-Tab costs Rs. 43,000 per patient, and this too at a sub-optimal dose of $1 \mathrm{~g}$ [15].

In fact, a recent trial found that the currently available poly valent Haffkine AVS was more effective than a monovalent AVS - Polonga-Tab that is prepared specifically against Sri Lankan Russell's viper venom [15].
The reported rates of adverse reactions to the polyvalent AVS used in Sri Lanka vary from about $30 \%$ to $68 \%$ [15], but only a small proportion $(5 \%$ to $10 \%$ ) of these reactions appear to be severe [15]. The overall rate of adverse reactions reported with the monovalent antivenom Polonga-Tab is $34 \%$ [15], and this too at what the authors themselves admit to being sub-optimal doses of the antiserum [15]. There is also concern about the possibility of more frequent adverse reactions with the use of a more effective higher initial dose of Polonga-Tab [15].

Proper Molecular studies of Sri Lankan snake venom and dose dependent studies were not done in Sri Lanka due to practical reasons. Assuming that the molecules of Indian snake are same as in Sri Lankan which may be doubtful and this may also have been contributed for the death in this case.

What doctors treating snakebite envenoming in Sri Lanka require is an effective, low reactogenic, cheap, and most importantly, a polyvalent AVS. Until that becomes available the currently available antisera imported from India, seem good enough [15].

\section{Conclusions}

At the end of the postmortem investigations, the COD was envenomation of neurotoxin due to cobra bite. The allegation of medical negligence may be challenged, because death could occur rapidly within 30 minutes to two hours in cobra bites and delay in transport may also have played a vital role especially being a rural area. Role of non-availability of species specific anti-venom and efficacy of common antisnake venom should be further studied before giving opinions regarding medical negligence.

This case highlights the need of further molecular studies and dose dependent studies in Sri Lankan snakebites, and preparation of Sri Lankan speciesspecific antivenom instead of Indian species-specific anti-venom.

\section{References}

1. Fernando R, Management of poisoning, $4^{\text {th }}$ Ed, The medical defence organization, 2011.

2. Guidelines for the management of snakebite in hospital. Ed. Fernando M, 2013. Expert Committee on Snakebite, Sri Lanka Medical Association Colombo.

3. Silva A, Gamlaksha D, Waidyaratne D. Medico-legal significance of the identification of offending snake in a fatal snake bite: a case report. Journal of Forensic 
and Legal Medicine. 2013 Nov; 20(8):9657.

4. SK Jindal, PS Shankar, Suhail Raoof, Dheeraj Gupta Textbook of Pulmonary and Critical Care Medicine Vols 1 and 2. New Delhi: Jaypee; 2011.p. 1699

5. Elapidae. https://en.wikipedia.org/ wiki/ Elapidae. accessed on 11.11.2015

6. Mohankumar P, Sivagurunathan C, Umadevi R. Study on the clinicoepidemiological profile and the outcome of snake bite victims in rural health centre in Kancheepuram District, Tamilnadu. International Journal of Pharma and Bio Sciences. 2015; 6(2):B544-550.

7. Kumar MR, Veeraprasad M, Babu PR, Kumar SS, Subrahmanyam BV, Rammohan $\mathrm{P}$, Srinivas M, et al. A retrospective review of snake bite victims admitted in a tertiary level teaching institute. Annals of African Medicine. 2014;13(2):76-80.

8. Drug Facts and Comparisons 2000. P 1499. https://books.google.lk/books?id=IWQ68q

NlKuAC\&q=Anti-snake+veno5m. Accessed on 14.11.2015.

9. Simpson ID, Norris RL. Snake Antivenom Product Guidelines in India: "The Devil is in the Details'. Wilderness and Environmental Medicine. 2007;18(3):163168.

10. Kularatne SA, Budagoda BD, Gawarammana IB, Kularatne WK. Epidemiology, clinical profile and management issues of cobra (Naja naja) bites in Sri Lanka: first authenticated case series. Transaction of the Royal Society of Tropical Medicine and Hygiene. 2009;103(9):924-30.

11. Bhat MK, Gowada TV. Purification and characterization of a myotoxic phospholipase A2 from Indian cobra (Naja naja naja) venom. Toxicon: Official journal of the international society of toxicology. 1989; 27(8):861-73.

12. Rudrammaji LM, Gowada TV. Purification and characterization of three acidic, cytotoxic phospholipases A2 from Indian cobra (Naja naja naja) venom. Toxicon: Official journal of the international society of toxicology. 1998;36(6):921-32.

13. De Silva A. Venomous snakes, their bites and treatment in Sri Lanka. In: Gopalakrishnakone P, Chou LM, editors. Snakes of medical importance. Singapore: National University Singapore; 1990. p. 479-546

14. Seneviratne SL, Opanayaka CJ, Ratnayake NS, Kumara KE, Sugathadasa AM, Weerasuriya N, Wickrama WA, Gunatilake $\mathrm{SB}$, de Silva HJ. Use of antivenom serum in snake bite: a prospective study of hospital practice in the Gampaha district. Ceylon Medical Journal.2000;45(2):65-8.

15. De Silva HJ, Fonseka MMD, Gunatilake SB, Kularatne SAM, Sellahewa KH. Antivenom for snakebite in Sri Lanka We need an effective, low reactogenic, affordable and polyvalent AVS. Ceylon medical journal. 2002;47(2):43-5. 